J3eA - Vol. 1 - 6 (2002).

DOI : 10.1051/bib-j3ea:2002006

\title{
Outils interactifs pour l'enseignement des convertisseurs électromécaniques : développement et mise en oeuvre
}

\author{
S. Labrique *, D. Grenier $* *$ et F. Labrique $* * *$ \\ Mis en ligne le 12 août 2002.
}

\begin{abstract}
Résumé
Cet article présente une initiative pédagogique, développée à la faculté des Sciences Appliquées de l'Université catholique de Louvain, pour l'enseignement des convertisseurs électromécaniques (machines électriques). Ce projet est basé sur un site internet, étroitement associé à un texte de base présenté sous forme de livre. Outre la description des différents types d'outils interactifs, et plus spécifiquement des animations (écrites en java) implémentées, l'article comporte une évaluation de ce type d'enseignement. Les réactions des étudiants ont été rassemblées, et constituent un instrument précieux pour mesurer l'impact de tels outils dans le processus d'apprentissage. Elles mettent également en exergue la nouvelle relation qui s'établit entre l'étudiant et l'enseignant : souci de profondeur de la part des étudiants, attention portée par l'enseignant à répondre aux questions propres des étudiants. Ces nouveaux outils interactifs sont développés en parallèle d'une pédagogie d'apprentissage par projet au cours duquel les étudiants mettent en pratique, par la production d'une oeuvre personnelle, les notions théoriques enseignées : réorganiser l'enseignement de base de l'électricité autour de ce dipôle nous semble ouvrir la voie à une formation moderne, véritablement efficiente, des sciences de l'ingénieur en général, et du génie électrique en particulier.

Ce projet a été développé avec le soutien du «Fonds de développement pédagogique » de l'Université catholique de Louvain.
\end{abstract}

Mots-clés. Électromécanique, convertisseurs, machines électriques, outils interactifs, multimédia, animations, applets JAVA, laboratoires virtuels.

(C) EDP Sciences, 2002.

Niveau de connaissances requis. Formation de base en physique et mathématiques (niveau bac +2 ). Niveau des étudiants. Second cycle, première année de la formation d'ingénieur électricien ou électromécanicien.

\footnotetext{
* Sophie Labrique est née le 7 novembre 1972 à Schaerbeek, Belgique. En 1995, elle a été diplômée ingénieur civil de la Faculté des Sciences Appliquées de l'Université catholique de Louvain, orientation architecture. De 1995 à 1999, elle a travaillé dans le bureau d'architecture et d'ingénierie ARCHI+I. Depuis 1999, elle travaille en tant qu'assistante de recherche au Laboratoire d'Électrotechnique et d'Instrumentation, à la réalisation d'ouvrages multimédia pour l'enseignement des convertisseurs électromécaniques et de la théorie de base des circuits électriques.
}

Ses domaines de recherche portent l'intégration des nouvelles technologies de la communication et de l'information dans l'enseignement du génie électrique aux ingénieurs. Elle est co-auteur de 5 communications ou articles sur le sujet.

Adresse postale : Université catholique de Louvain, Laboratoire d'Électrotechnique et d'Instrumentation, 3, place du Levant, B-1348 Louvain-La-Neuve, Belgium.

email : Sophie.Labrique@lei.ucl.ac.be 
** Damien Grenier est né à Rouen (France) en 1965. Il a été étudiant de l' École Normale Supérieure de Cachan (France) où il a reçu le doctorat en Ingénierie Électrique en 1994. De 1994 à 1996, il a effectué un séjour postdoctoral de recherche à l'École de Technologie Supérieure de Montréal (Canada). En 1996, il a rejoint en tant que Professeur le laboratoire d'Électrotechnique et d'Instrumentation (LEI) de la Faculté des Sciences Appliquées de l'Université catholique de Louvain (UCL).

Ses domaines de recherche sont la conception, la modélisation et la commande digitale des convertisseurs électromécaniques. Il est co-auteur d'un livre sur les convertisseurs électromécaniques et d'environ 40 articles ou communications scientifiques.

Adresse postale : Université catholique de Louvain, Laboratoire d'Électrotechnique et d'Instrumentation, 3, place du Levant, B-1348 Louvain-La-Neuve, Belgium.

email : grenier@lei.ucl.ac.be

*** Francis Labrique est né à Maurage, Belgique, le 16 mars 1946. Il est ingénieur civil électricien et docteur en sciences appliquées de l'Université catholique de Louvain. Depuis 1970, il est membre du Laboratoire d'Electrotechnique et d'Instrumentation de la Faculté des Sciences Appliquées de la même université, où il est actuellement professeur.

Ses domaines de recherche sont l'électronique de puissance et la commande de systèmes électromécaniques (en particulier pour l'aéronautique). Il est co-auteur de 6 livres dans le domaine et d'environ 140 articles ou communications scientifiques.

Adresse postale : Université catholique de Louvain, Laboratoire d'Électrotechnique et d'Instrumentation, 3, place du Levant, B-1348 Louvain-La-Neuve, Belgium.

email : labrique@lei.ucl.ac.be

\section{Introduction}

Cet article présente une initiative pédagogique, développée à la faculté des Sciences Appliquées de l'Université catholique de Louvain, pour l'enseignement des convertisseurs électromécaniques (machines électriques).

Il décrit les divers outils interactifs que nous avons développés et aborde, à travers l'évaluation faite auprès des étudiants, la véritable plus-value que nous en avons recueillie dans notre pratique d'enseignants.

En premier lieu, nous présenterons les motivations qui ont guidé ce projet et nous énumérerons les raisons qui nous ont décidé à investiguer les opportunités offertes par le multimédia.

Nous préciserons le concept développé, qui associe étroitement un livre et un site internet.

Nous détaillerons ensuite les différents types d'outils interactifs que nous avons développés, et examinerons l'apport spécifique de chacun d'entre eux dans le processus d'apprentissage de l'étudiant.

Nous nous attarderons sur quelques-uns de ces outils, et plus spécifiquement sur des animations (écrites en java) représentatives des différentes possibilités d'illustration des concepts théoriques.

Pour chacune d'entre elles :

- nous préciserons l'objectif pédagogique que nous nous étions fixé,

- nous décrirons le synopsis didactique dans lequel elle s'insère

- et nous examinerons l'interactivité qu'elle offre à l'étudiant.

Enfin, suite à la mise en oeuvre des premiers laboratoires virtuels, les réactions des étudiants ont été rassemblées. Ces réactions consistuent un instrument précieux pour l'évaluation de ces laboratoires et la mesure de l'impact de tels outils interactifs dans le processus d'apprentissage. Elles mettent également en exergue la nouvelle relation qui s'établit entre l'enseignant et l'apprenant, dont nous ne sommes pas loin de penser qu'elle représente une part importante, si ce n'est essentielle, de la plus-value apportée par ces outils multimédia à notre enseignement. 


\section{Les motivations du projet}

Faire comprendre aux étudiants les mécanismes qui sous-tendent la conversion électromécanique au sein des machines électriques n'est pas chose aisée.

Comme tout ce qui relève du domaine du génie électrique, cela exige tout d'abord de la part des étudiants un niveau d'abstraction beaucoup plus poussé que dans d'autres disciplines. En génie mécanique par exemple, les enseignements peuvent aisément faire appel à des expériences vécues par tout un chacun. Les notions élémentaires de position, de vitesse, de forces, qu'elles soient de gravité, centrifuges, d'attraction..., sont des notions intuitives qu'il suffit (même si cela n'est pas toujours simple) de qualifier et de quantifier correctement pour écrire le modèle d'un dispositif mécanique. Nul n'a jamais vu en revanche une charge, un courant ou un potentiel électrique autrement que par les effets qu'ils produisent. Il en va de même pour les notions de champs électriques et magnétiques ou de flux. Quand ces grandeurs évoluent de plus dans le temps et dans l'espace, comprendre comment toutes ces grandeurs physiques peuvent interagir pour créer des efforts mécaniques est loin d'être immédiat.

Se rendre au laboratoire peut être d'une grande aide, parce qu'il permet de tester en temps réel comment un convertisseur réagit aux variations des grandeurs à ses accès mécaniques et électriques. Malheureusement, pour des raisons de temps et de sécurité, les étudiants ne sont généralement pas autorisés à effectuer tous les essais qui leur seraient nécessaires pour clarifier leur compréhension de la théorie. Plus encore, les essais en laboratoire ne peuvent être effectués que sur des machines de faible puissance, dont les caractéristiques ne sont pas toujours représentatives de celles de puissance moyenne ou élevée.

L'utilisation d'outils multimédia interactifs peut aider l'enseignant dans sa démarche. Ainsi, par exemple :

- Les figures animées sont irremplaçables pour visualiser la manière dont les grandeurs physiques internes des convertisseurs électromécaniques évoluent dans le temps et dans l'espace.

- Alors qu'il est usuel dans un ouvrage sur support papier ou au tableau lors d'un cours, de tracer les courbes ou diagrammes qui permettent de caractériser le fonctionnement d'un convertisseur pour un jeu de paramètres bien choisi, le support informatique permet à l'étudiant de voir comment ceux-ci sont affectés (ou non) par une modification des paramètres constructifs de la machine ou de son alimentation. Cette possibilité s'applique en particulier à tous les diagrammes vectoriels (phasoriels) utilisés habituellement en électrotechnique pour étudier les convertisseurs électromécaniques à champ tournant.

- Les outils de simulation permettent la réalisation de laboratoires virtuels. Ces laboratoires, s'ils ne prétendent pas remplacer la confrontation indispensable avec la réalité, permettent d'offrir aux étudiants plus d'occasion de manipuler plus souvent ces objets difficiles à comprendre que sont les convertisseurs électromécaniques et ainsi de se les approprier. Il leur est même possible d'apprendre en faisant des erreurs, en « cassant » virtuellement des machines, choses que pour des raisons de coût et surtout de sécurité il est impossible de leur laisser faire dans un laboratoire réel. Enfin et surtout, cela leur permet de manipuler des machines dans des gammes de puissance qu'aucune installation didactique ne leur permettra jamais d'atteindre et de constater à cette occasion qu'un moteur de quelques watts ne se comporte pas toujours de la même façon qu'un moteur de plusieurs centaines de kilowatts.

- Les questionnaires à choix multiple sont un moyen utile à l'étudiant pour s'auto-évaluer et s'assurer qu'il a effectivement compris la matière. Ils lui sont aussi une occasion de se poser des questions qui sous une apparence parfois triviale cachent des problèmes plus compliqués qu'il n'y paraît à première vue. 


\section{Le concept : l'association d'un livre et d'un site internet}

Notre site internet héberge, nous l'avons évoqué, des outils interactifs efficaces pour apprendre et comprendre la conversion électromécanique.

Un site internet ne peut cependant pas tout. Dès qu'une explication demande de passer par un texte un peu long (plus d'un ou deux écrans), le premier réflexe des étudiants est d'imprimer ce texte, le support papier s'avérant plus confortable que l'écran d'ordinateur.

En outre, en rassemblant dans un livre l'ensemble des notions théoriques, on peut mieux structurer leur présentation et mettre en évidence les liens qui existent entre elles que lorsqu'elles se trouvent dessiminées entre des exemples, des illustrations et des laboratoires virtuels.

Le concept développé associe donc étroitement un texte contenant les références théoriques, présenté sous forme de livre [1], et un site internet [2].

\section{Un livre}

Le livre «Électromécanique : convertisseur d'énergie et actionneurs » [1] présente la théorie des convertisseurs électromécaniques sous une forme relativement compacte (320 pages). En effet, comme l'écrit M. le Professeur Guy Séguier dans sa préface, son association au site mutimédia interactif permet de réserver cet ouvrage à la présentation de l'essentiel, les développements usuellement traités dans les traités classiques mais jugés moins indispensables étant reportés sur le site. Son objet ainsi décanté, il peut présenter dans les meilleurs conditions la théorie de la conversion électromécaniques, son application aux divers types de machines, les caractéristiques de celle-ci et la mise œuvre de leurs possibilités par leur alimentation et leur commande.

\section{Un site intermet}

À ce livre est associé un site internet (www.electromecanique.net) destiné à illustrer, visualiser et permettre de mieux appréhender les concepts théoriques (Fig. 1).

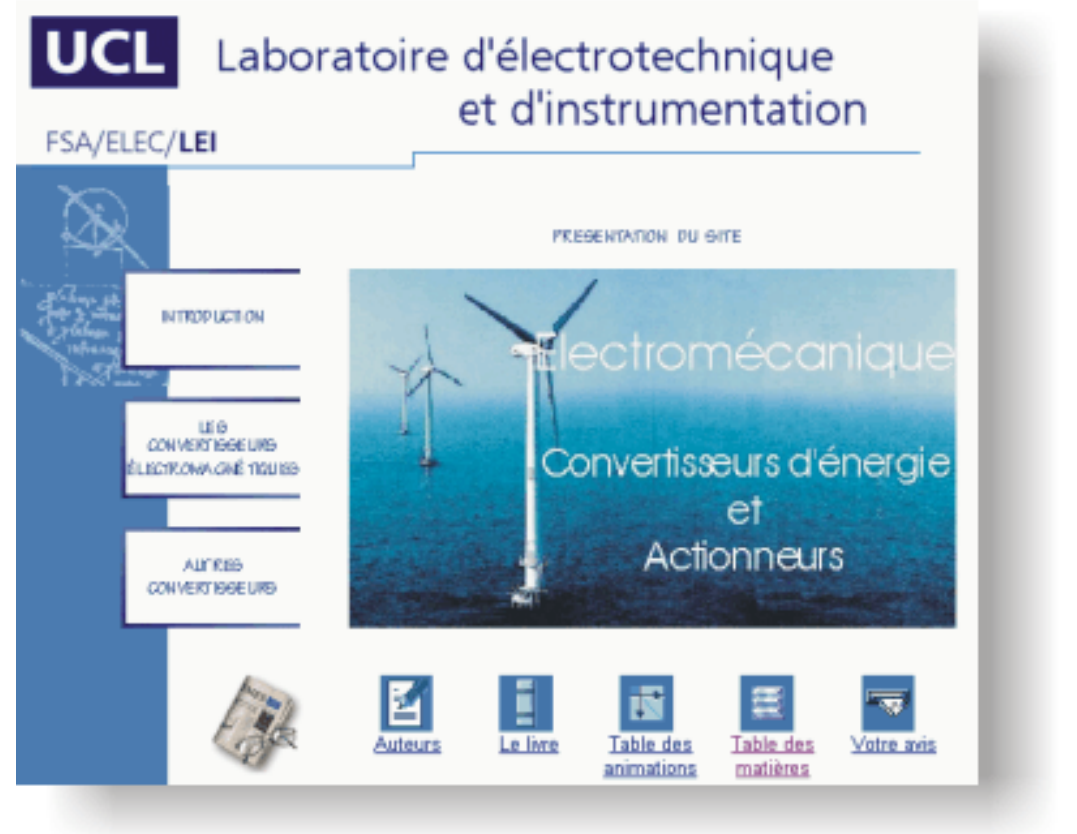

Fig. 1. Page d'accueil du site www.electromecanique.net.

Pour faciliter la liaison entre le livre et le site, l'organisation du site suit rigoureusement le plan du livre, et se structure suivant les différents chapitres (Fig. 2). 


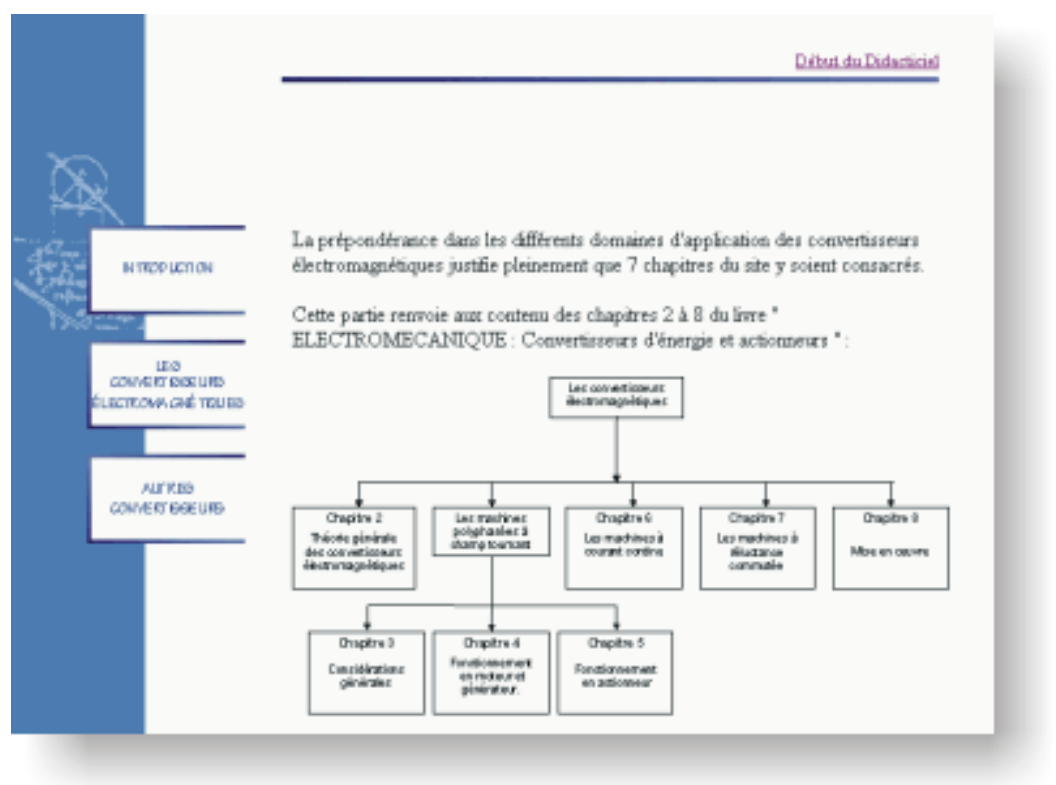

Fig. 2. Plan du site.

Pour chaque chapitre du livre, le site propose une série de rubriques (Fig. 3), qui offrent à l'étudiant différentes approches interactives pour tester sa compréhension de la matière :

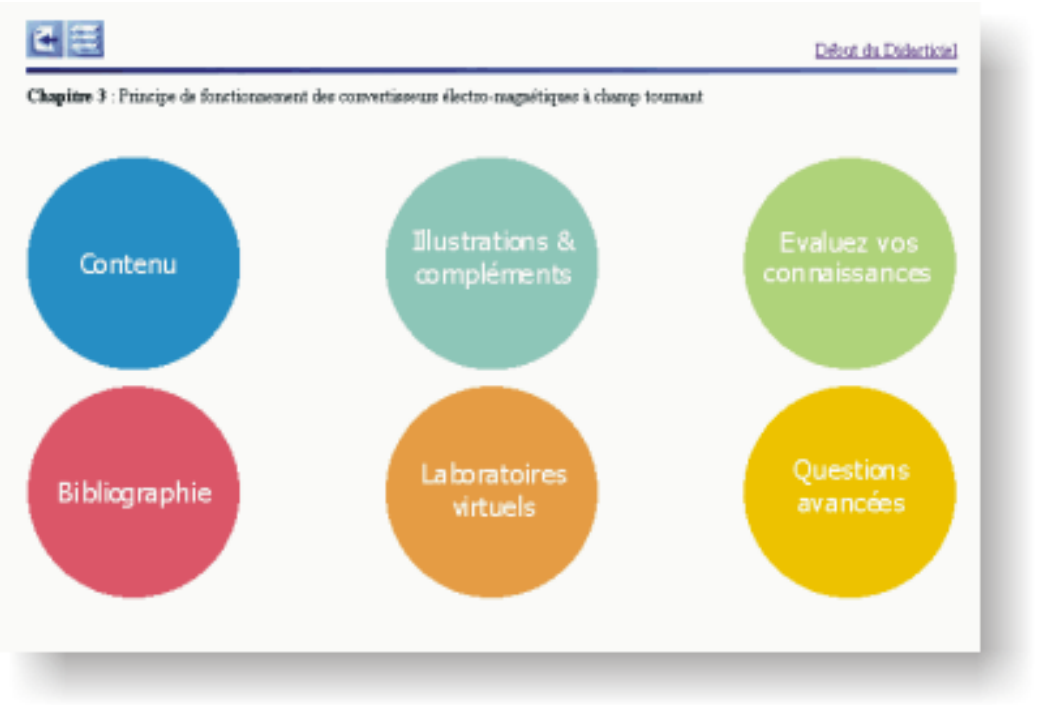

Fig. 3. Page d'accueil d'un chapitre.

- La rubrique « Illustrations et compléments » donne accès à une liste d'exposés qui illustrent et complètent le contenu du chapitre, au moyen d'animations, de photos et/ou de courtes séquences vidéo.

Exemple d'illustration : la notion de champ tournant http://www.lei.ucl.ac.be/multimedia/Convertisseurs/chapitre_345/chapitre_3/theorie/5_theorie/0_cours. $\mathrm{htm}$

- La rubrique «Laboratoires virtuels » propose des exercices résolus, des expériences virtuelles, ou la simulation du fonctionnement des convertisseurs électromécaniques sous différentes conditions. Chaque laboratoire contient l'énoncé du problème et une série de questions, des boutons d'aide, et un cheminement progressif vers la réponse (illustrée par des graphes animés ou des simulations) et sa démonstration.

Exemple de laboratoire virtuel : le moteur réluctant oscillant http://www.lei.ucl.ac.be/multimedia/Convertisseurs/chapitre_2/labos/MoteurReluctant/enonce.htm 
- La rubrique « Testez vos connaissances » permet à l'étudiant, par le biais de questionnaires à choix multiple, de vérifier et mesurer son niveau de connaissance et de compréhension de la matière traitée dans le chapitre.

Exemple d'un questionnaire à choix multiple

http://www.lei.ucl.ac.be/multimedia/Convertisseurs/chapitre_2/qcm/Question.htm

- Les rubriques « Bibliographie » et «Questions avancées » référencent des articles et références bibliographiques qui permettent l'approfondissement de certains points de la matière.

\section{L'apport spécifique des différents outils interactifs}

Nous allons à présent détailler les différents types d'outils interactifs, et examiner l'apport spécifique de chacun d'entre eux dans le processus d'apprentissage de l'étudiant.

\section{La navigation}

La navigation permet de présenter les exercices et laboratoires sous une forme progressive. À partir de l'énoncé,

- une aide peut éventuellement être apportée à l'étudiant, indiquant les hypothèses qu'il serait raisonnable de poser, les équations ou théorèmes utiles à la résolution du problème,

- la définition de certains termes est rappelée dans le glossaire,

- la réponse est donnée, éventuellement illustrée de graphes animés ou de simulations. La démonstration n'est pas encore visible à ce stade, pour permettre à l'étudiant de reprendre son raisonnement et de vérifier ses calculs s'il obtient un résultat différent,

- la démonstration de la réponse permet à l'étudiant de comparer son raisonnement avec celui (ou ceux) que nous proposons.

- une vérification de la réponse est parfois proposée : un résultat expérimental ou un calcul par éléments finis permet a posteriori la validation des hypothèses simplificatrices et/ou en montre les limites. 


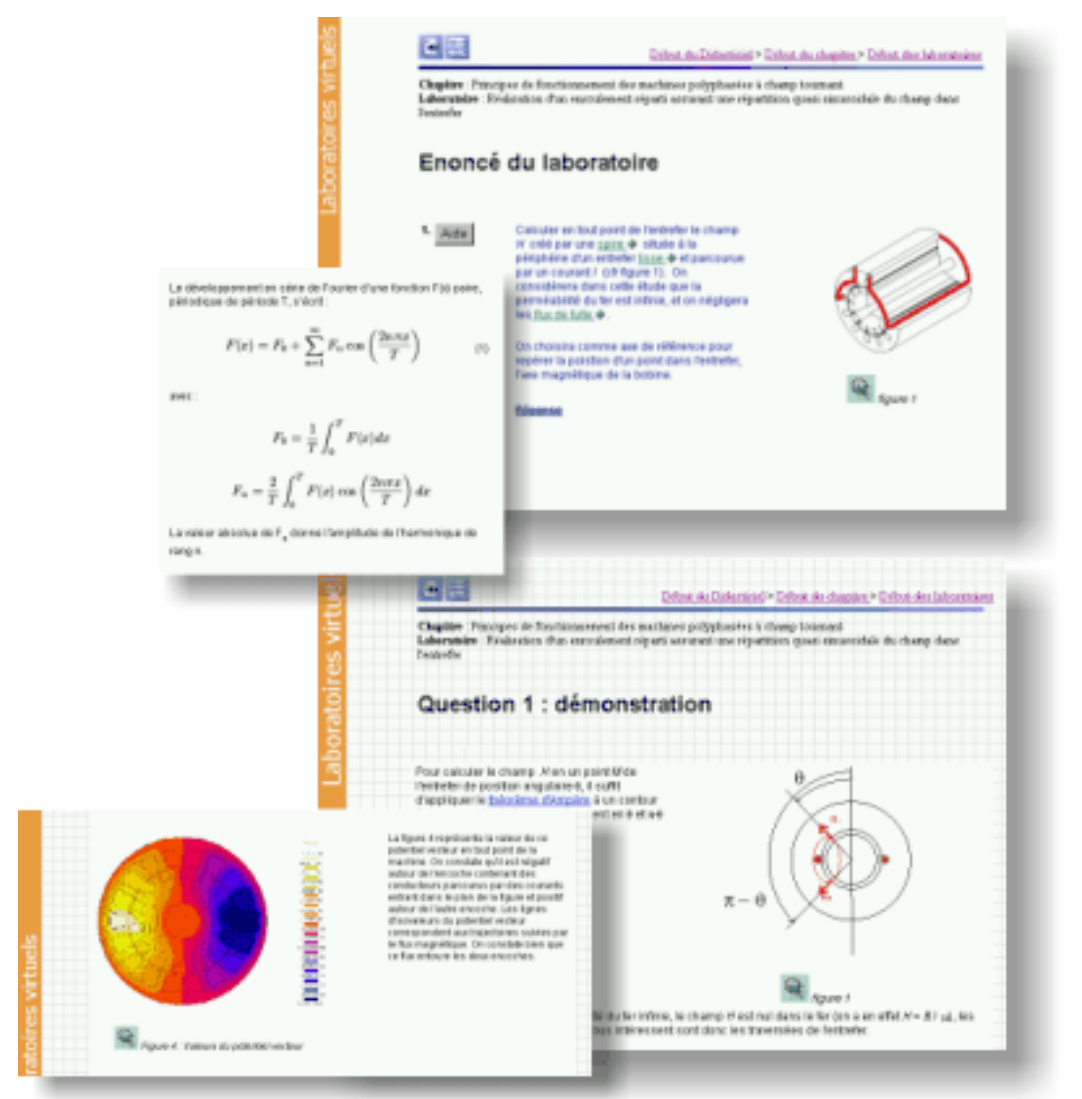

Fig. 4. Présentation des exercices et des laboratoires virtuels sous une forme progressive.

Visualiser un laboratoire virtuel sur le site www.electromecanique.net

http://www.lei.ucl.ac.be/multimedia/Convertisseurs/chapitre_345/chapitre_3/labos/1_labo/home.htm

\section{Les photos etlervidion}

Les photos et les vidéos permettent de visualiser l'aspect technologique en montrant différentes machines électriques, et les étapes de fabrication de celles-ci. Elles offrent la possibilité de voir «à quoi ressemblent » les machines électriques.

Les vidéos offrent en outre l'avantage de permettre à l'étudiant d'appréhender plus facilement les structures tridimensionnelles.

\section{Les questionnaires is choix multiple}

Les questionnaires à choix multiple permettent à l'étudiant, par le biais d'une correction automatique, de vérifier et mesurer son niveau de connaissance et de compréhension de la matière. Ils fournissent également un moyen de se préparer à l'examen. 


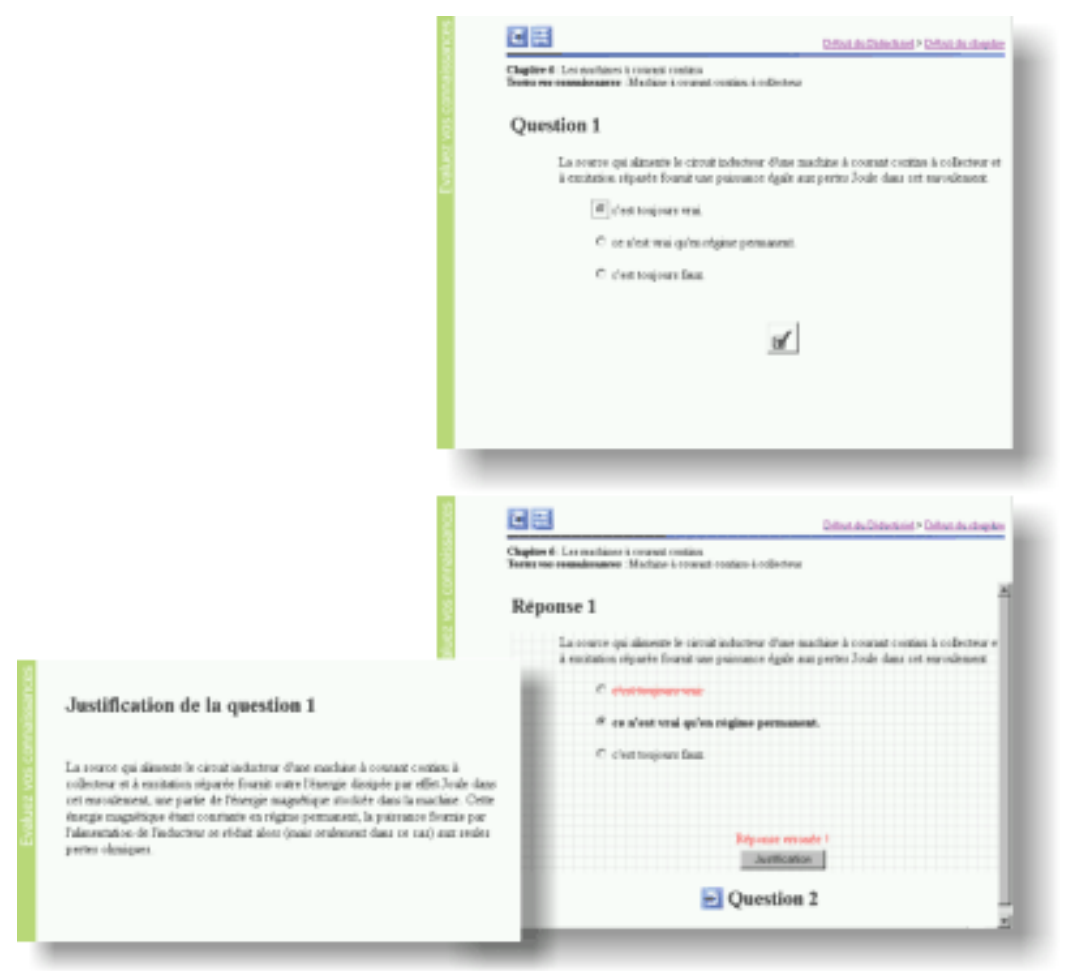

Fig. 5. Questionnaire à choix multiple, et correction automatique.

Visualiser un QCM sur le site www.electromecanique.net

http://www.lei.ucl.ac.be/multimedia/Convertisseurs/chapitre_345/chapitre_5/qcm/questionnaire_1/Question.htm

\section{Les animations java}

Nous consacrons une rubrique entière à l'implémentation des animations (écrites en java), car celles-ci représentent, nous en sommes convaincus, la partie la plus importante, mais surtout la plus innovante de notre travail.

La notion d'interactivité y est en effet maximale, puisque grâce à ces animations, l'étudiant peut en effet visualiser « en temps réel » l'évolution d'une grandeur, mesurer l'influence d'un jeu de paramètres qu'il a configuré lui-même, manipuler virtuellement les convertisseurs, apprendre en faisant des erreurs, manier des machines dans des gammes de puissance les plus extrêmes, etc. 


\section{Les animations java}

Les animations java permettent d'aborder différents thèmes allant des aspects constructifs des convertisseurs électromécaniques à leurs conditions de fonctionnement dans différents domaines d'application.

\section{Notion de champtourmant}

\section{Objectif pédagogique :}

En guise d'illustration du chapitre 3 du livre, portant sur les principes de fonctionnement des convertisseurs électromagnétiques à champ tournant, nous voulons illustrer la notion de champ tournant (voir l'illustration correspondante sur le site www.electromecanique.net :

http://www.lei.ucl.ac.be/multimedia/Convertisseurs/chapitre_345/chapitre_3/theorie/5_theorie/0_cours. $\mathrm{htm}$ ) et permettre la visualisation des phénomènes de variations électromagnétiques spatio-temporelles, en relation avec la conversion d'énergie dans les machines à courant alternatif.

\section{Synopsis didactique :}

Cet exposé, présenté sous forme d'une succession d'écrans, regroupe une série de graphes animés (Animation 1). Après avoir montré comment évolue le champ d'entrefer d'un enroulement alimenté par un courant sinusoïdal, ces animations montrent graduellement comment le champ d'entrefer créé par trois enroulements décalés spatialement de $120^{\circ}$, alimentés par des courants déphasés temporellement de $120^{\circ}$ conduit à la production d'un champ tournant.

\section{Interactivité :}

Ces graphes animés permettent la visualisation simultanée des variations dans le temps et dans l'espace du champ d'entrefer. L'étudiant peut dès lors « voir » que la somme de trois champs pulsés déphasés spatialement et temporellement de $120^{\circ}$ les uns par rapport aux autres donne un champ tournant.

Animation 1 : Visualisation du champ tournant (visualiser cette animation sur le site www.electromecanique.net :

http://www.lei.ucl.ac.be/multimedia/Convertisseurs/chapitre_345/chapitre_3/theorie/5_theorie/7_cours.htm)

\section{Aspects constructifs}

\section{Objectif pédagogique :}

Dans le cadre d'un laboratoire virtuel portant sur la réalisation d'un enroulement réparti assurant une répartition quasi sinusoïdale du champ dans l'entrefer (voir le laboratoire correspondant sur le site www.electromecanique.net :

http://www.lei.ucl.ac.be/multimedia/Convertisseurs/chapitre_345/chapitre_3/labos/1_labo/home.htm), nous voulons permettre à l'étudiant de comprendre comment les paramètres constructifs (le « design ») influencent les caractéristiques de la machine et comment construire une machine qui s'approche du modèle idéal décrit dans l'illustration précédente sur la notion de champ tournant.

\section{Synopsis didactique :}

Dans ce laboratoire, l'étudiant est progressivement amené à calculer et à visualiser le champ d'entrefer et les coefficients de Fourier des harmoniques d'espaces de ce champ :

- dans le cas d'une spire ;

- dans le cas d'une bobine (constituée de $n$ spires);

- dans le cas de deux bobines, dont il peut faire varier l'angle d'étalement ;

- dans le cas de $m$ bobines, dont il peut faire varier le nombre et l'angle d'étalement. 
Animation 2 : Influence de l'étalement des conducteurs sur le contenu harmonique du champ d'entrefer (visualiser cette animation sur le site www.electromecanique.net :

http://www.lei.ucl.ac.be/multimedia/Convertisseurs/chapitre_345/chapitre_3/labos/1_labo/51_reponse.htm).

Une comparaison des résultats obtenus par le calcul simplifié avec ceux fournis par un calcul par éléments finis permet de vérifier la validité de l'étude effectuée.
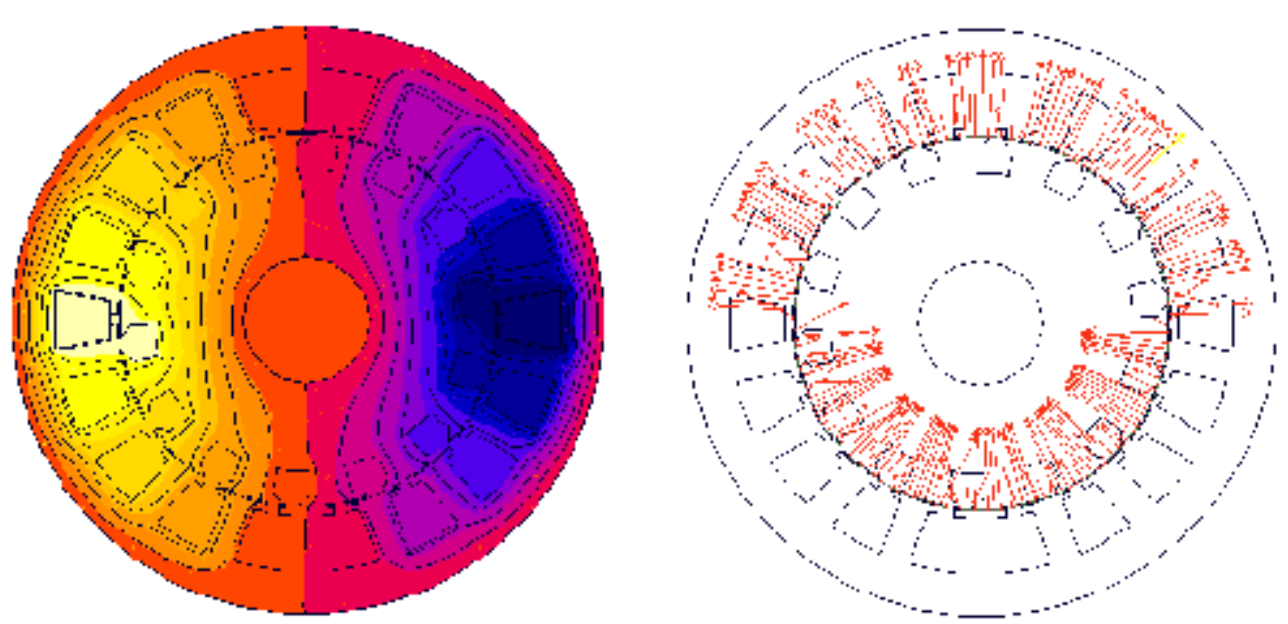

Fig. 6. Validation du calcul simplifié par comparaison avec un calcul par éléments finis (cas où $m=1$ ).

En fin de laboratoire, l'étudiant est amené à comparer les valeurs des coefficients de Fourier des différentes harmoniques, dans le cas d'une machine diphasée et dans celui d'une machine triphasée, dont les enroulements seraient étalés au maximum $\left(60^{\circ}\right.$ pour la machine triphasée, $90^{\circ}$ pour la machine diphasée). Il est ainsi amené à conclure que la machine triphasée avec une alimentation triphasée en courant est toujours meilleure qu'une machine diphasée parce que :

- une alimentation triphasée en courants sans homopolaire fait disparaître la composante harmonique de rang 3 du champ total créé par les trois enroulements,

- un étalement de $60^{\circ}$ est proche de la valeur de $72^{\circ}$ qui annule la composante harmonique de rang 5 de ce champ.

\section{Interactivité :}

Pour chacun de ces cas, nous avons développé des interfaces interactives (Animation 2) qui permettent de faire varier les valeurs de différents paramètres constructifs, comme l'angle d'étalement ou le nombre de bobines, et de visualiser instantanément leur influence sur le champ et les coefficients de Fourier des harmoniques d'espaces de ce champ.

\section{Couplage surleséseaud'une machine synchrone}

\section{Objectif pédagogique :}

Dans le cadre de l'étude du fonctionnement en moteur ou en générateur des machines triphasées à champ tournant qui font l'objet du chapitre 4, le laboratoire virtuel consacré à l'étude de la machine synchrone (voir l'énoncé correspondant sur le site www.electromecanique.net :

http://www.lei.ucl.ac.be/multimedia/Convertisseurs/chapitre_345/chapitre_4/labos/2_labo/enonce.htm) connectée au réseau à travers une impédance de liaison a pour but de permettre à l'étudiant de visualiser

- comment on réalise le couplage d'un alternateur au réseau,

- puis comment on règle ses échanges de puissances active et réactive avec le réseau. 


\section{Synopsis didactique :}

L'étudiant est amené dans une première étape à réaliser les opérations qui permettent de synchroniser un alternateur sur le réseau. Pour cela, nous avons développé une animation (Animation 3) émulant le fonctionnement d'un synchronoscope. Il est ensuite amené à voir comment on peut régler les échanges de puissances active et réactive avec le réseau, en agissant sur la puissance mécanique fournie par le moteur d'entraînement et le courant inducteur. L'étudiant peut aussi, se faisant, aller jusqu'à faire (virtuellement) décrocher la machine.

Il peut voir comment l'utilisation d'un régulateur de tension permet d'augmenter la limite de stabilité.

\section{Interactivité :}

L'animation 3 permet une manipulation virtuelle de la machine. La visualisation des opérations de réglage du point de fonctionnement (Animation 4) s'effectue à l'aide d'un diagramme vectoriel animé, qui évalue en fonction des paramètres de réglage (puissance mécanique et courant inducteur, ou puissance mécanique et tension aux bornes de la machine).

Animation 3 : Synchronisation de la machine sur le réseau (visualiser cette animation sur le site www.electromecanique.net :

http://www.lei.ucl.ac.be/multimedia/Convertisseurs/chapitre_345/chapitre_4/labos/2_labo/3_enonce.htm\#animat ion1).

Animation 4 : Réglage du point de fonctionnement d'un alternateur connecté au réseau (visualiser cette animation sur le site www.electromecanique.net :

http://www.lei.ucl.ac.be/multimedia/Convertisseurs/chapitre_345/chapitre_4/labos/2_labo/3_enonce.htm\#animat ion2).

\section{Mise en oeuvre et évaluation de ces outils interactifs}

Certains laboratoires virtuels ont d'ores et déjà été testés auprès de groupes d'étudiants, et leurs réactions ont été rassemblées.

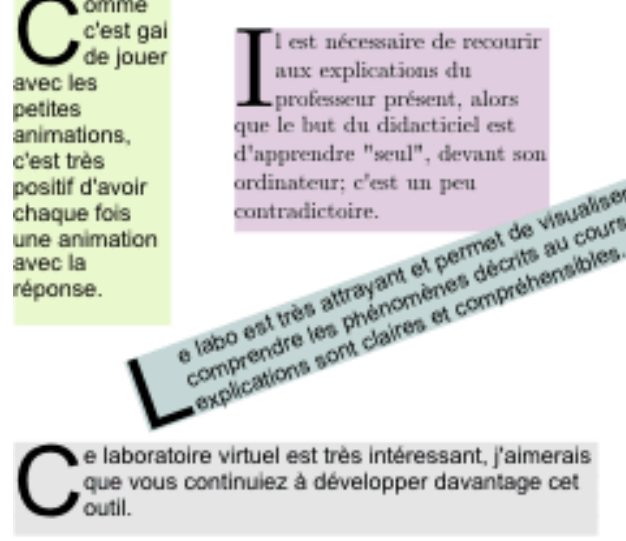

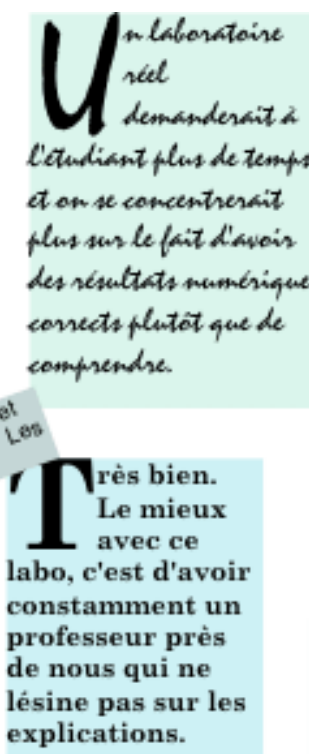

Fig. 7. Extraits choisis des réactions des étudiants (commentaires libres).
T y a un meilleur rapport "compétences acquises / temps consacré" que pour les laboratoires réels.

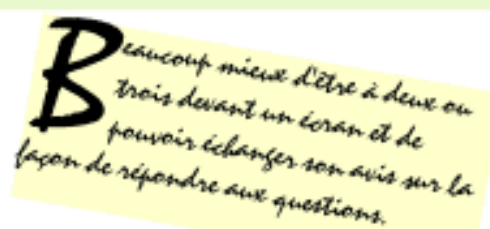

\section{7 rès intóressant on ce qui concerne és animations mais manque de temps si on vent te faire conscionciensement.}

Difficultés à se crncentrer sur un écrun

$\square$ ermet d'être plus actif qu'à une séance d'exercices car à trois sur un pc, on n'est jamais bloqué par un problème tandis qu'en séance, un assistant ne sait pas faire face aux questions de 30 étudiants à la fois. 


\section{Efficacité pédazogique}

Principaux résultats du sondage effectué auprès des étudiants :

- $\quad 83 \%$ estiment avoir beaucoup appris ;

- $66 \%$ des étudiants estiment avoir appris plus qu'à un cours ;

- $\quad 53 \%$ des étudiants estiment avoir appris plus qu'à une séance d'exercices ou un laboratoire réel ;

Ceci permet d'épingler que les outils interactifs renforcent l'efficacité d'une séance de travaux pratiques. Ce rendement accru semble s'expliquer par plusieurs facteurs que nous avons relevés dans leurs réactions :

1. L'ordinateur permet une approche progressive, « à son propre rythme ».

2. L'attitude de l'étudiant est plus active, et plus orientée vers la compréhension que vers le calcul.

3. La mise à disposition d'un corrigé de l'exercice permet à l'enseignant qui encadre la séance de se consacrer aux questions de fond relatives à la compréhension de la matière.

\section{Les apports spécifiquerdumultimédia}

Les animations sont plébiscitées par $85 \%$ des étudiants qui les apprécient comme l'apport multimédia essentiel, et considèrent qu'elles leur sont très utiles pour une compréhension plus intuitive de la matière.

Ils jugent ces animations « bien pensées », « très claires », « très utiles », interactives, et estiment qu'elles leur permettent de mieux saisir les nuances, mais surtout de « visualiser » les concepts.

\section{L'e-learning remplacera-t-illeprofessens?}

La possibilité d'utiliser ce site internet pour l'auto-apprentissage ou la formation à distance est rejetée par nos étudiants

- $\quad$ qui soulignent l'importance du travail en groupe, avec la possibilité d'échanges à 2 ou 3 étudiants qu'il permet ;

- qui jugent essentielle la présence d'un enseignant dans la salle, car l'enseignant peut répondre aux questions qui sont suscitées par l'exercice, mais s'éloignent du spectre des réponses implémentées dans le laboratoire virtuel.

Cependant, le nombre de connections au site (d'accès libre) par des utilisateurs autres que nos propres étudiants (environ $1 / 3$ des accès) montre que même si elle est moins efficace, l'utilisation du site à distance reste utile. Les réactions de certains de ces utilisateurs montrent également que le recours au livre de référence [1] n'est pas indispensable, et peut être remplacé par d'autres références sur le même sujet [3] [4].

A épingler également, la demande d'un support papier pour la lecture des pages Web qui contiennent beaucoup de texte : d'où, a posteriori, la confirmation de notre option de départ d'un livre associé à un site. 


\section{Conclusion}

En écoutant les étudiants, nous pouvons conclure, au terme de cette première expérience, qu'ils estiment que le développement de ce type d'outils multimédia leur apporte un véritable « plus » dans l'apprentissage de la matière. L'essentiel de la plus-value se situe d'ailleurs dans l'interactivité proposée par ces outils qui consolident le processus d'apprentissage, sans se substituer ni au support écrit (texte de référence), ni aux cours ou aux séances encadrées de travaux pratiques. En ce sens, la migration brute du contenu traditionnel d'un cours vers les nouvelles technologies n'a pas d'intérêt en tant que tel.

Ces outils deviennent par ailleurs le support d'une nouvelle relation qui s'établit entre l'enseignant et l'étudiant :

- Les étudiants sont beaucoup plus actifs qu'à une séance d'exercices classique : l'aspect « ludique » des animations, la navigation à travers le site, suscitent une participation accrue des étudiants.

- On remarque un souci de profondeur de la part des étudiants : libérés de leur rôle de "gratte-papier », les étudiants concentrent leurs efforts à une compréhension plus profonde, à une analyse beaucoup plus fine, ce que traduit la qualité des questions posées à l'enseignant. Par rapport à un laboratoire réel, les étudiants se focalisent sur la compréhension des phénomènes, sur les compétences à acquérir, au-delà du fait d'obtenir « des résultats numériques corrects ».

- l'enseignant également, libéré de la tâche répétitive de transmettre la réponse aux questions de l'énoncé, peut concentrer son attention à répondre aux questions propres des étudiants, suscitées ou non par l'exercice.

Une question demeure en suspens : cette approche virtuelle ne risque-t-elle pas d'éloigner les étudiants du monde physique ? Pour éviter cela, nous développons, en parallèle de ces outils interactifs, une pédagogie d'apprentissage par projet, au cours duquel les étudiants mettent en pratique, par la production d'une oeuvre personnelle, les notions théoriques enseignées. Ce type d'enseignement, implémenté dès le premier cycle de leur formation d'ingénieur (réforme « Candi $2000 »$ ) [5], est déjà présent dans le cursus de nos étudiants électromécaniciens en 4ème année (niveau bac +4 ), qui conçoivent et réalisent des robots mobiles [6] et [7]. Il sera encore renforcé dès la 1ère année technique (niveau bac +3 ) dans le cadre d'une réforme de l'enseignement de base de l'électricité décidée par notre faculté.

Coaliser ces nouveaux outils interactifs et l'apprentissage du projet nous semble donc ouvrir la voie à une formation moderne, véritablement efficiente, des sciences de l'ingénieur en général, et du génie électrique en particulier.

\section{Références bibliographiques}

[1] H. Buyse, D. Grenier, F. Labrique et E. Matagne, Électromécanique : Convertisseurs d'énergie et actionneurs (Dunod, 2001).

[2] adresse du site : www.electromecanique.net

[3] G. Séguier et F. Notelet, Électrotechnique industrielle, 2ème édition (Édition Technique \& Documentation, Lavoisier, 1994).

[4] M. Juffer, Électromécanique, Traité d'Electricité, volume 11 (Presses polytechniques et universitaires romandes, 1995).

[5] E. Aguirre, C. Jacqmot, E. Milgrom, B. Raucent, A. Soucisse, Ch. Trullemans et C. Vander Borght, Devenir ingénieur par apprentissage actif, Pédagogie par le projet, Brest 27-29 juin 2001.

[6] D. Grenier, P. Sente, P. Fisette et B. Raucent, La conception et la réalisation de robots mobiles comme éléments fédérateurs d'une formation en mécatronique, Actes du Colloque sur l'Enseignement des Technologies et des Sciences de l'information et des Systèmes (CESTIS-EEA'99), 295-298, Montpellier (France), Novembre 1999.

[7] D. Grenier, P. Fisette et B. Raucent, Fédérer des activités pédagogiques pour constituer un projet intégré en mécatronique : compte rendu d'innovation, Didaskalia 16 (Avril 2000) 163-178. 